\title{
Acoustic Liner Drag: Measurements on Novel Facesheet Perforate Geometries
}

\author{
Brian M. Howerton ${ }^{1}$ and Michael G. Jones. ${ }^{2}$ \\ NASA Langley Research Center, Hampton, VA, 23681
}

\begin{abstract}
Interest in characterization of the aerodynamic drag of acoustic liners has increased in the past several years. This paper details experiments in the NASA Langley Grazing Flow Impedance Tube to quantify the relative drag of several perforate-over-honeycomb liner configurations at flow speeds of $M=0.3$ and 0.5 . Various perforate geometries and orientations are investigated to determine their resistance factors using a static pressure drop approach. Comparison of these resistance factors gives a relative measurement of liner drag. For these same flow conditions, acoustic measurements are performed with tonal excitation from 400 to $3000 \mathrm{~Hz}$ at source sound pressure levels of 140 and $150 \mathrm{~dB}$. Educed impedance and attenuation spectra are used to determine the impact of variations in perforate geometry on acoustic performance.
\end{abstract}

\section{Nomenclature}

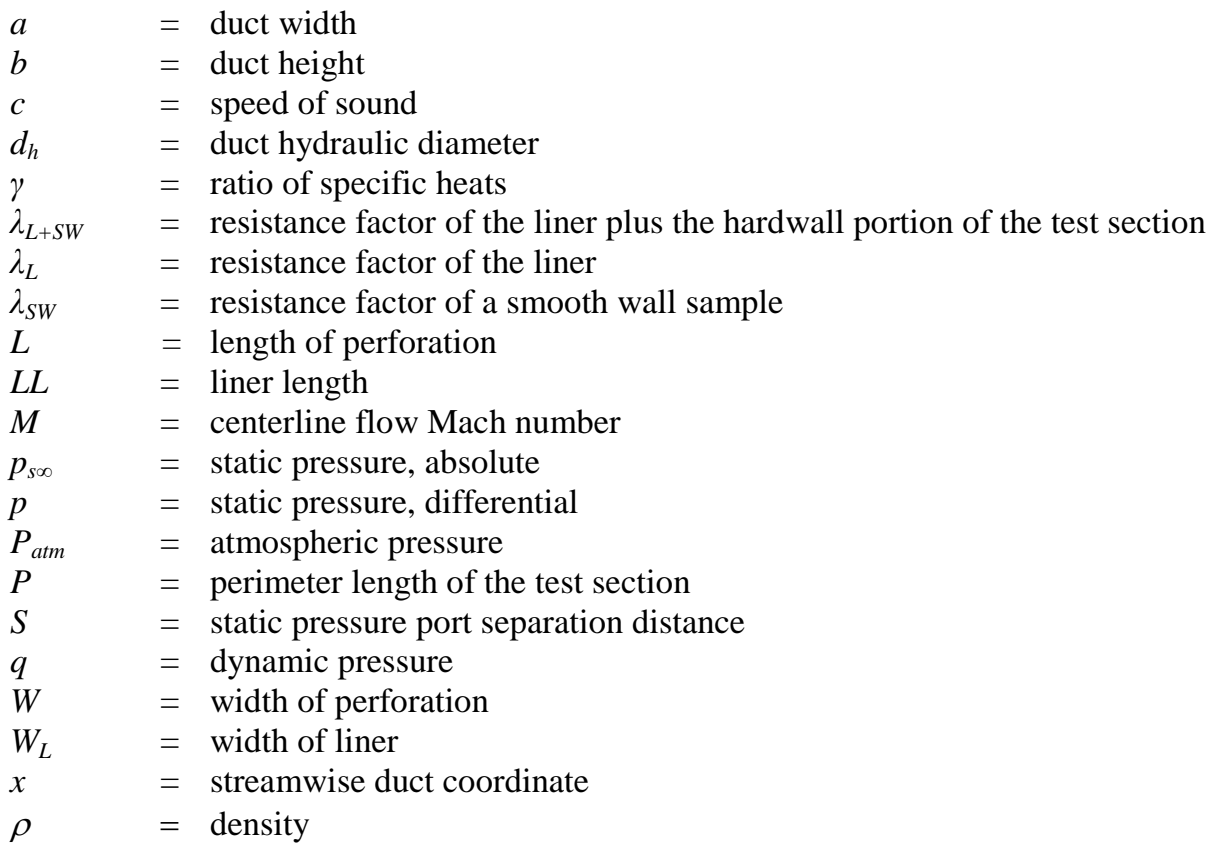

\section{Introduction}

Tn the past, the aerodynamic drag imparted by placing acoustic liners in an aircraft engine was tolerated as a necessary penalty in order to meet the required noise levels. It is generally accepted that such liners inevitably increase drag relative to a smooth surface. ${ }^{1}$ Research by Drouin has also shown that liner drag can be influenced by the ambient acoustic field. ${ }^{2}$ Continued pressure on manufacturers to reduce engine emissions and fuel burn provides the motivation to understand and reduce drag associated with engine nacelle liners. Furthermore, new aircraft

\footnotetext{
${ }^{1}$ Research Scientist, Structural Acoustics Branch, MS 463, Senior Member AIAA.

${ }^{2}$ Senior Research Scientist, Structural Acoustics Branch, MS 463, Associate Fellow AIAA.
} 
propulsion concepts (open-rotor, distributed electric) may lead to airframe designs where external liners (Fig. 1) are required to meet community noise goals. ${ }^{3}$

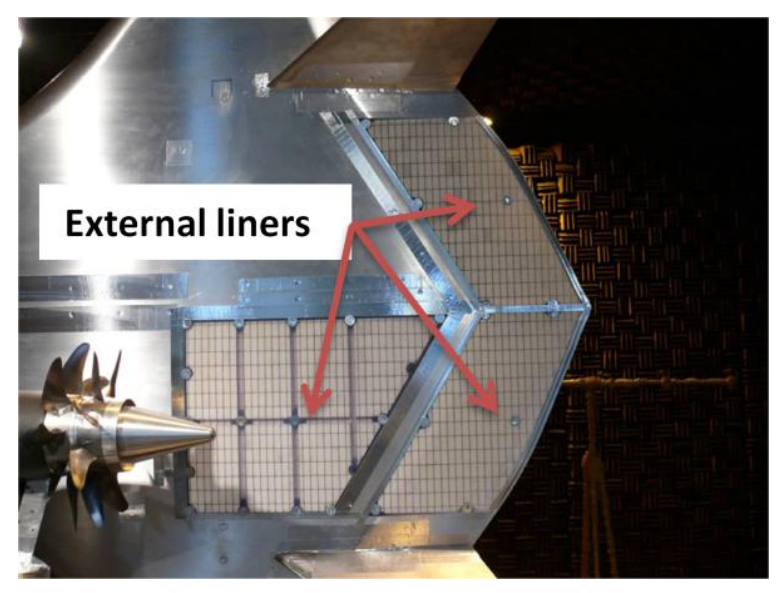

Figure 1. External liners on an Open-Rotor powered, Blended Wing-Body concept aircraft.

NASA is committed to developing a suite of technologies to aggressively reduce aircraft fuel consumption and noise. To that end, creating a low-drag acoustic liner would be an important contribution. The majority of acoustic liners developed for production aircraft engines use a facesheet perforated with round holes to provide the necessary porosity for use in a liner. An approach from Nikuradse ${ }^{4}$ allows for calculation of a resistance factor $(\lambda)$ based on the static pressure drop within a lined duct. Howerton and Jones ${ }^{5}$ used this approach to show that reducing the perforate hole diameter produced a reduction in $\lambda$ (and thus the drag) for a typical liner design (Fig. 2).
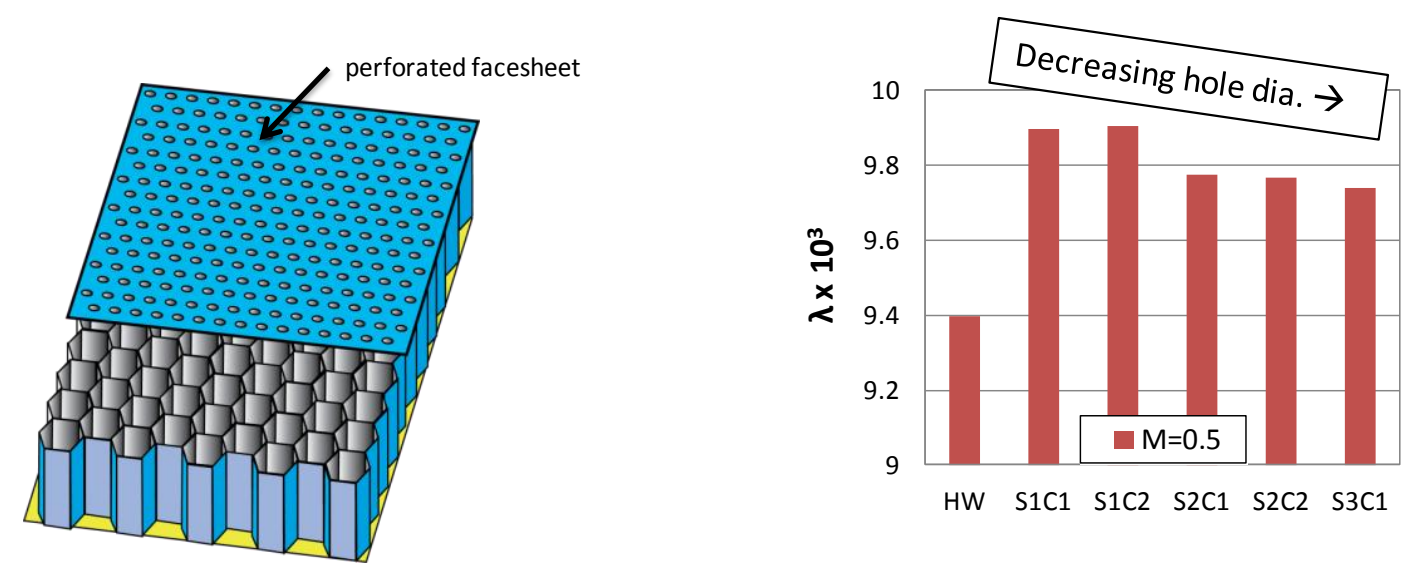

Figure 2. Conventional liner construction and the effect of perforate hole dia. on $\lambda$.

Changing the perforate geometry and orientation, relative to the flow, may create a configuration with improved drag performance. Advances in three-dimensional (3-D) printing allow for parametric studies of these concepts to develop an empirical database of geometric variations and are the first step towards formulation of analytical liner drag models.

The purpose of the current investigation is to evaluate the effects of perforate geometry and orientation on the drag with a number of novel facesheet perforate configurations. Each liner is tested in the NASA Langley Research Center Grazing Flow Impedance Tube, and the resultant acoustic and aerodynamic responses are compared to (1) determine which designs provide reduced drag, and (2) determine whether this drag reduction has an impact on the acoustic performance of the liner. Details are provided on the static pressure drop method used to compute $\lambda$ along with a description of the liner samples tested. Later sections show these results for various perforate geometries with 
and without acoustic excitation. Data is also presented on liner acoustic performance focusing on the educed impedances and resulting attenuation.

\section{Liner Drag Measurements}

For this investigation, the drag of each configuration relative to a smooth wall will be determined by measuring differences in the static pressure drop along the duct wall opposite of the liner sample. This method can be applied to small ducts with fully-developed, turbulent flow and is similar to Nikuradse's approach when studying roughness in pipes. ${ }^{4}$ With the static pressure data and selected flow parameters, one can compute the duct resistance factor, $\lambda$ (also known as the 'friction factor'), given by the following:

$$
\lambda=\frac{d p}{d x} \frac{d_{h}}{q}
$$

using the hydraulic diameter of the flow duct for $\mathrm{d}_{\mathrm{h}}$ :

$$
d_{h}=\frac{2 a b}{a+b}
$$

and the compressible form for q:

$$
q=\frac{\gamma}{2} p_{s \infty} M^{2}
$$

The non-dimensional nature of $\lambda$ allows the static pressure data to be normalized, taking out the run-to-run effects of slightly varying duct Mach number and static pressure. Note that $\lambda$ encompasses the sum of both the skin friction and pressure components of drag. Thus, any effects of the liner cavity are also included, thereby differentiating this method from others that are solely measuring skin friction. The results of these calculations can be used to provide a relative measurement of drag between liner configurations.

\section{Experiment}

The experimental investigation involves testing of eleven liner facesheet and two smooth wall configurations in the GFIT. The facesheet samples are created using a 3-D printing process and placed over a common liner core. An aluminum smooth wall sample (SW) with no perforations is included to provide a reference baseline. For each configuration, a static pressure survey is performed along the length of the GFIT. Simultaneously, a measurement of the axial static pressure drop across the liner is made using higher accuracy instrumentation for computation of the liner resistance factor.

\section{A. Facesheet Construction}

The majority of the sheets designed for this experiment have a constant 8 percent open area (POA) and a sheet thickness of $0.762 \mathrm{~mm}$. One sheet was built to a higher porosity of $14 \mathrm{POA}$ while another was substantially thicker at $7.62 \mathrm{~mm}$ to give a thickness-to-hole diameter (T/D) ratio of 10. Multiple perforate geometries are evaluated. In addition, a 3-D printed smooth wall sample (Resin SW) with no perforations is included to evaluate any inherent effects of the manufacturing process and material. Figure 3 provides sketches of the test geometries. A summary of the key parameters for each perforation type is given in Table 1. Several round-hole perforates were constructed since this is the most common geometry in current use and is the simplest to manufacture. These configurations are identified with white labels in the table. In addition to the Conventional sample with straight holes, variations in hole angle (45 and $60 \mathrm{deg}$ from horizontal) and orientation (with, against and perpendicular to the flow direction) are investigated. Note that setting orientation with or against the flow is achieved by rotating the angled-hole facesheets to reverse the leading and trailing edges. Alternate geometries of slots with the long axis both parallel and perpendicular to the flow, and diamond-shaped perforations are also considered. Based on data from a study by Hwang $^{6}$ which indicated that increasing the facesheet thickness may result in lower drag, an example with a higher thickness-to-hole diameter ratio (T/D 10) was built as well. The facesheets were 3-D printed from photopolymer resin using a stereolithography (SLA) process with the flow surface sanded to ensure a smooth finish. 

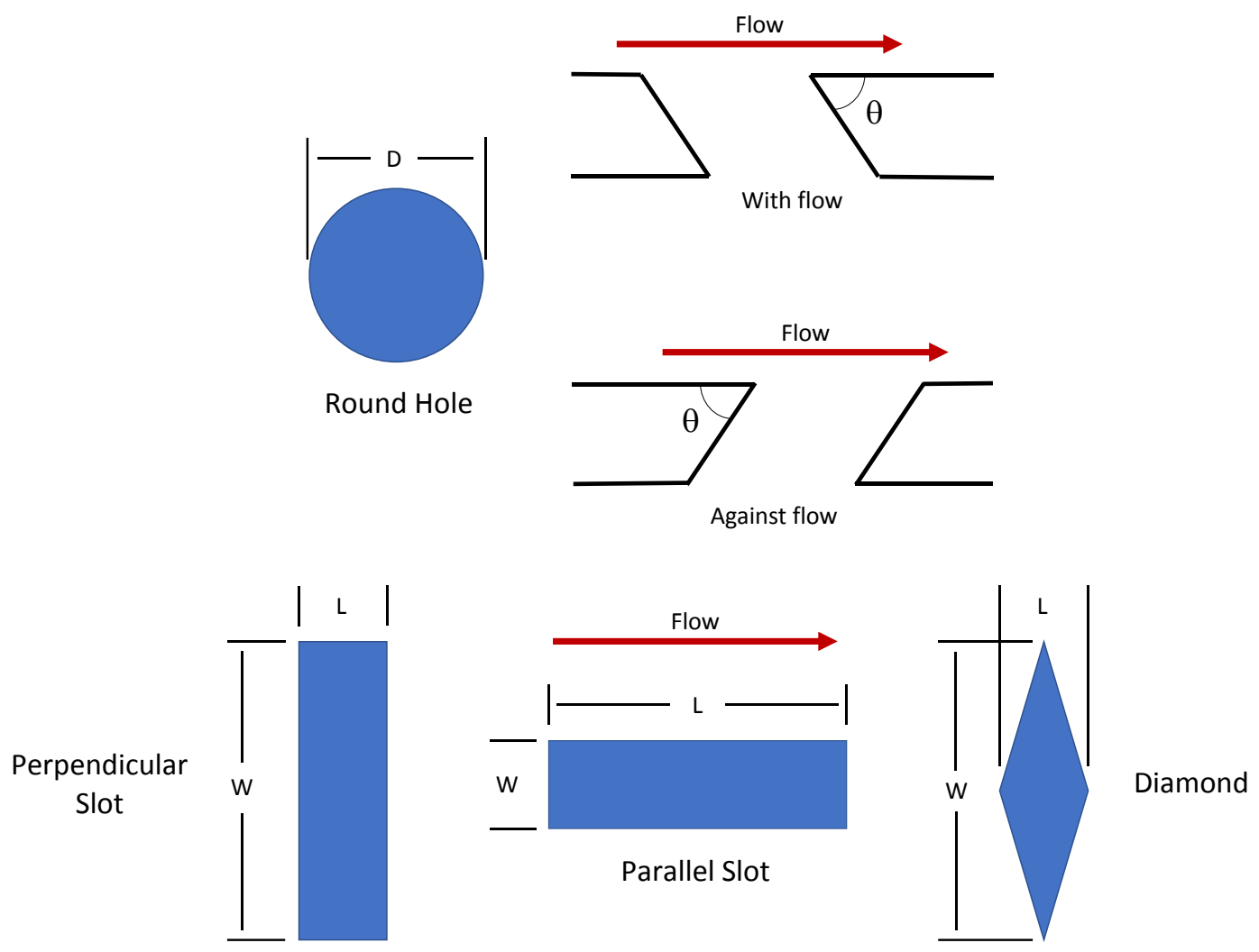

Figure 3. Facesheet perforate geometry.

\begin{tabular}{|c|c|c|c|c|c|c|c|c|}
\hline & Conventional & $\begin{array}{c}60 \mathrm{deg} \\
\text { w/flow }\end{array}$ & $\begin{array}{c}60 \mathrm{deg} \\
\text { a/flow }\end{array}$ & $\begin{array}{c}45 \mathrm{deg} \\
\text { w/flow }\end{array}$ & $\begin{array}{c}45 \mathrm{deg} \\
\text { a/flow }\end{array}$ & $\begin{array}{c}60 \mathrm{deg} \\
\text { p/flow }\end{array}$ & $\begin{array}{c}\text { High } \\
\text { POA }\end{array}$ & $\begin{array}{c}\text { High } \\
\text { T/D }\end{array}$ \\
\hline $\mathrm{D}(\mathrm{mm})$ & 0.762 & 0.762 & 0.762 & 0.762 & 0.762 & 0.762 & 1.016 & 0.762 \\
\hline$\theta(\mathrm{deg})$ & 90 & 45 & 45 & 60 & 60 & 60 & 90 & 90 \\
\hline
\end{tabular}

\begin{tabular}{|c|c|c|c|}
\hline & $\begin{array}{c}\text { Parallel } \\
\text { Slot }\end{array}$ & $\begin{array}{c}\text { Perpendicular } \\
\text { Slot }\end{array}$ & Diamond \\
\hline $\mathrm{L}(\mathrm{mm})$ & 2.032 & 0.508 & 0.508 \\
\hline $\mathrm{W}(\mathrm{mm})$ & 0.508 & 2.032 & 2.032 \\
\hline$\theta(\mathrm{deg})$ & 90 & 90 & 90 \\
\hline
\end{tabular}

Table 1. Facesheet perforate dimensions. 


\section{B. Liner Core Construction}

Each facesheet was mounted onto a metallic liner core with a cavity depth of $38.1 \mathrm{~mm}$. The liner core is from a previous investigation that was repurposed for this experiment. To allow for rapid changes of liner configuration, the facesheets are not bonded to the core structure but clamped by their long edges as part of installation in the test rig. Note that the core is constructed for the full length of the GFIT test window $(614.4 \mathrm{~mm})$ while the facesheets are only $460.8 \mathrm{~mm}$ long. An aluminum filler blank was fabricated to cover the remaining portion of the core. Figure 4 shows a typical arrangement with the filler blank (left) and the facesheet (right) overlaid on the core.

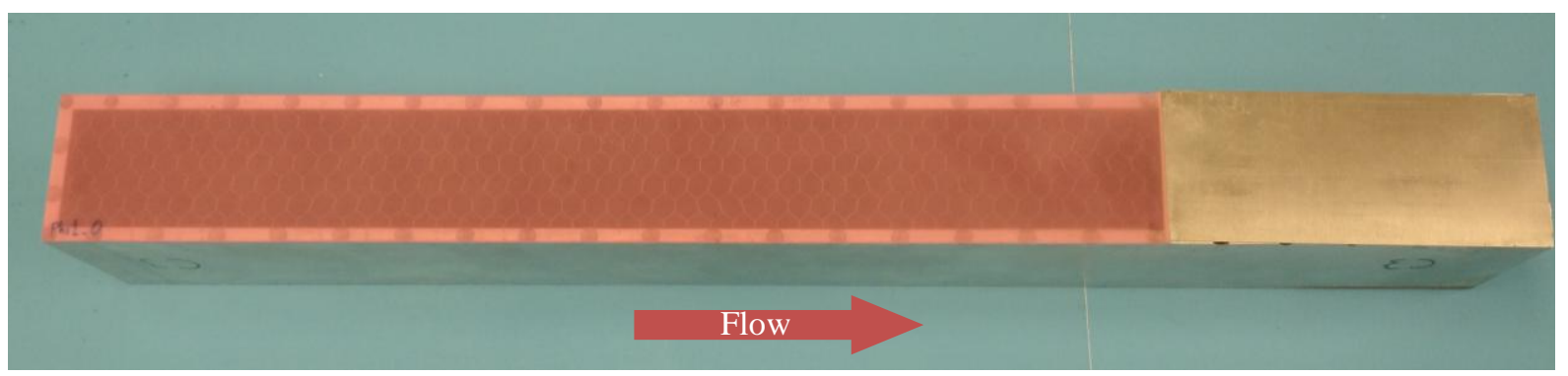

Figure 4. Liner sample facesheet and filler blank overlaid on core cavitiy with internal honeycomb.

\section{Grazing Flow Impedance Tube (GFIT)}

The Grazing Flow Impedance Tube (GFIT) is a unique facility originally constructed to determine the acoustic characteristics of noise reduction treatments (acoustic liners) for aircraft jet engine nacelles and nozzles. The facility is a small wind tunnel with a $50.8 \mathrm{~mm}$ by $63.5 \mathrm{~mm}$ rectangular cross section. The flow path (Fig. 5) is a straight duct with a 12-driver upstream acoustic source section, interchangeable lengths of blank duct, a test section holding the liner sample along the upper wall of the duct and an array of 95 measurement microphones leading to a 6-driver downstream source section and anechoic terminating diffuser. Pressurized, heated air is supplied to the entrance of the GFIT while a vacuum system is used at the duct exit to 'pull' the flow out of the tube. This arrangement allows for the static pressure at the test section to be near ambient at all flow velocities while also creating an adiabatic wall condition. In its current configuration, samples can be tested at grazing flow velocities from 0 to Mach 0.6 and sound pressure levels up to $150 \mathrm{~dB}$ for the frequency range between 400 and $3000 \mathrm{~Hz}$.

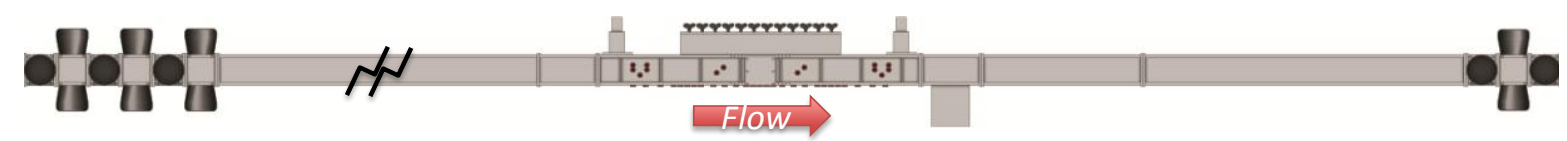

Figure 5. Sketch of the NASA Langley Grazing Flow Impedance Tube (GFIT).

This investigation also makes use of the array of 80 static pressure ports located along the lower wall of the duct to measure the axial pressure distribution. Pressures from these ports are simultaneously sampled by a series of transducers with a $+/-17 \mathrm{kPa}$ range and $0.05 \% \mathrm{FS}$ accuracy with a fixed sample rate of $100 \mathrm{~Hz}$. A plot of the axial pressure distribution in the test section for the hardwall case is shown in Fig. 6. Two ports, one located near the entrance and the other located near the exit of the test section (separated axially by $1.07 \mathrm{~m}$ ) are also connected to a high-accuracy, differential pressure gauge to measure the static pressure drop between these two locations. This gauge samples at a much slower rate $(\sim 10 \mathrm{~Hz})$ but with its smaller $0-6900 \mathrm{~Pa}$ range and $0.01 \% \mathrm{FS}$ accuracy, measurement uncertainty is reduced by a factor of 12.5. A sketch of the test section is included above the plot showing the relative location of the liner and the ports used to compute the static pressure drop $(\Delta \mathrm{p})$. The highresolution measurement points are designated as Port 37 and Port 59, respectively. 


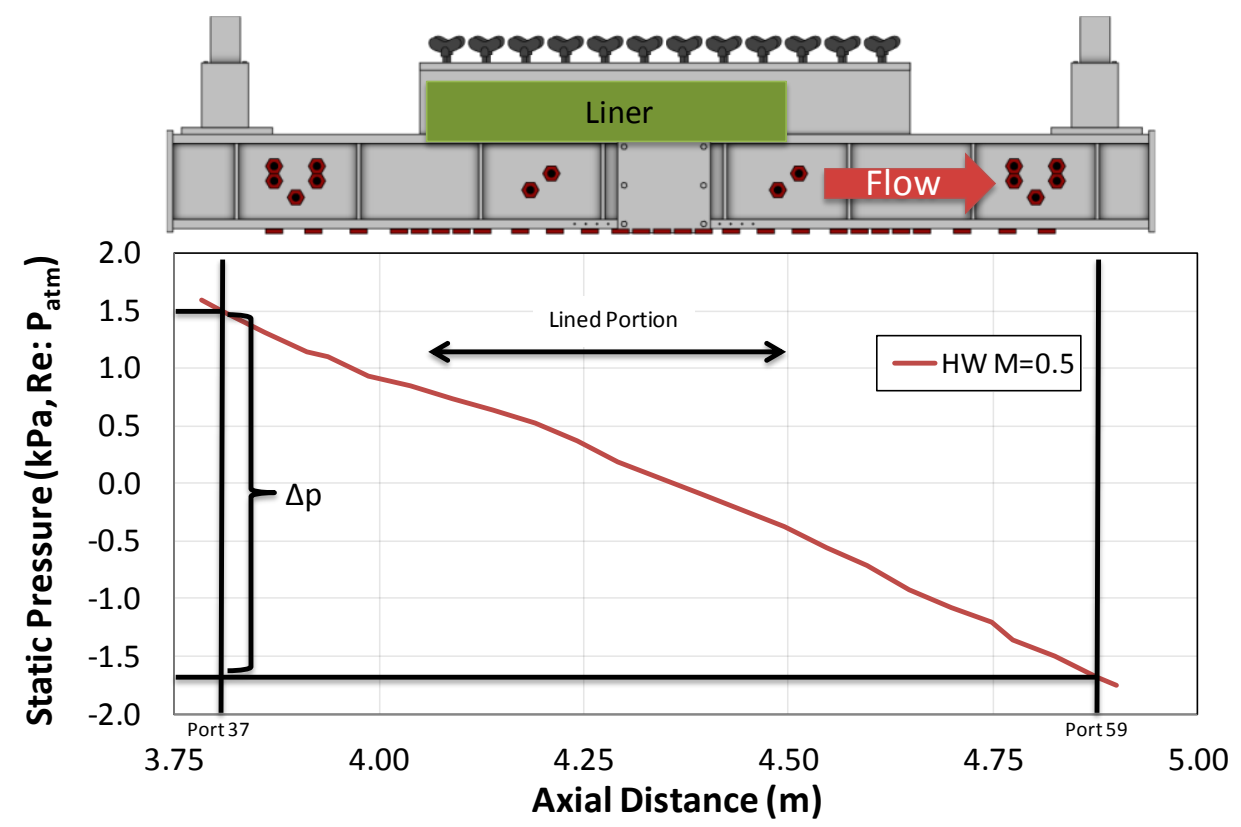

Figure 6. GFIT Test Section Static Pressure Distribution, Hardwall sample, $M=0.5$.

\section{Measurement Process}

Following the method set forth by Howerton and Jones ${ }^{4}$, averaged static pressure measurements were made for each configuration with no acoustic excitation at $\mathrm{M}=0.3$ and 0.5 . For each data set, 1000 readings from each static port were made over a nominal $40 \mathrm{sec}$ period then averaged to give one measurement per port. Simultaneously, similar data were acquired from the high-accuracy gauge to provide the static pressure drop across the length of the liner. For all cases, the target Mach number was held to a tolerance of $+/-0.002$ while static pressure in the test section is set within +/-130 Pa. Tunnel conditions, including average Mach number and static pressure, are also recorded to allow computation of $\lambda$ from Eq. (2). Use of a non-dimensional coefficient like $\lambda$ provides a benefit by normalizing the static pressure data. This normalization reduces the variability of the results, allowing comparison of data from different flow runs where static pressure and Mach number differences (albeit small) can affect the raw $\Delta p$ measurements. An example of this variation is shown in the left plot of Fig. 7 with a graph of $\Delta \mathrm{p}$ measurements from the smooth wall case at nominally $\mathrm{M}=0.5$. The existence of a relationship between Mach number and $\Delta \mathrm{p}$ is readily apparent. Computation of $\lambda$ from this data results in the plots shown in the right plot of Fig. 7.
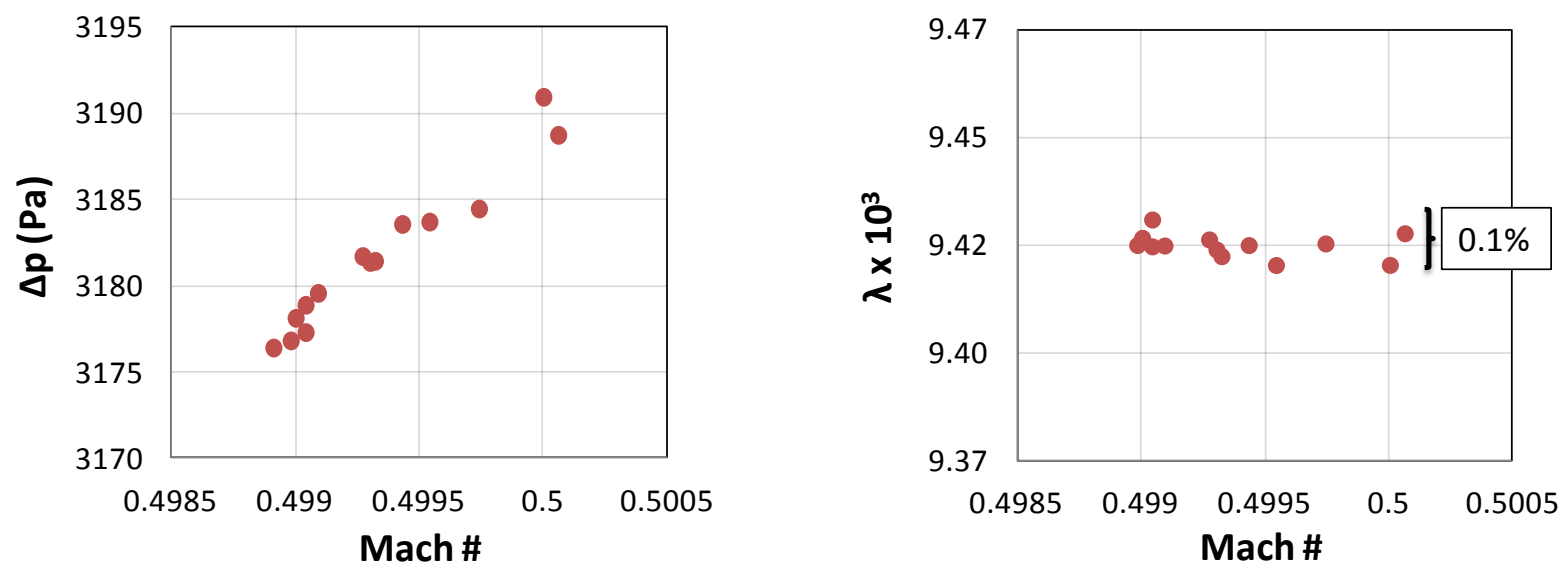

Figure 7. Liner $\Delta p$ measurements and corresponding values of $\lambda$, Smooth wall sample, $M=0.5$.

The calculated values of $\lambda$ are independent of the small Mach number changes and variability of the results about the mean is nominally $0.1 \%$, indicating excellent repeatability. Comparisons with other flow speeds show variability 
decreasing with increasing Mach number, since $\Delta \mathrm{p}$ increases while the accuracy of the pressure gauge is fixed as a percentage of its range. Note that values of $\lambda$ derived from GFIT pressure measurements cannot be directly related to values of Darcy's friction factor commonly found on a Moody chart. Only a portion of the duct surface is lined and, depending upon $\mathrm{M}$ and the axial location of the test section, the flow may not be fully developed.

If one assumes that the contribution of each portion of the duct to the measured value of lambda is proportional to the surface area, the resistance factor of the lined portion can be determined using the following relation:

$$
\lambda_{L+S W}=\frac{P-W_{L}}{P} \lambda_{S W}+\frac{W_{L}}{P}\left(\frac{S-L L}{S} \lambda_{S W}+\frac{L L}{S} \lambda_{L}\right)
$$

Solving for $\lambda_{\mathrm{L}}$ gives:

$$
\lambda_{L}=\frac{\frac{P \lambda_{L+S W}-\left(P-W_{L}\right) \lambda_{S W}}{W_{L}}-\left(\frac{S-L L}{S}\right) \lambda_{S W}}{\frac{L L}{S}}
$$

Tonal acoustic excitation was additionally used for $\mathrm{M}=0.3$ and $\mathrm{M}=0.5$ for frequencies between 400 and $2800 \mathrm{~Hz}$ (200 Hz increments) at a target sound pressure level (SPL) of 140 and $150 \mathrm{~dB}$ (re: $20 \mu \mathrm{Pa}$ ). It is important to note that, for certain combinations of frequency and Mach number, the higher SPL was not achieved but was usually at least $6 \mathrm{~dB}$ greater than the lower target. Static pressure measurements were performed simultaneously with the acoustic surveys to evaluate the effect of acoustic excitation on liner drag. Acoustic measurements were performed to allow for comparison of liner impedances educed using the Straightforward Method of Watson ${ }^{7}$.

\section{Results and Discussion}

\section{A. Static Pressure Measurements}

Figure 8 shows the liner resistance factor $\left(\lambda_{\mathrm{L}}\right.$, computed from Eq. (6)) for the eleven facesheet and two smooth wall configurations tested at Mach numbers of 0.3 and 0.5 with no sound.

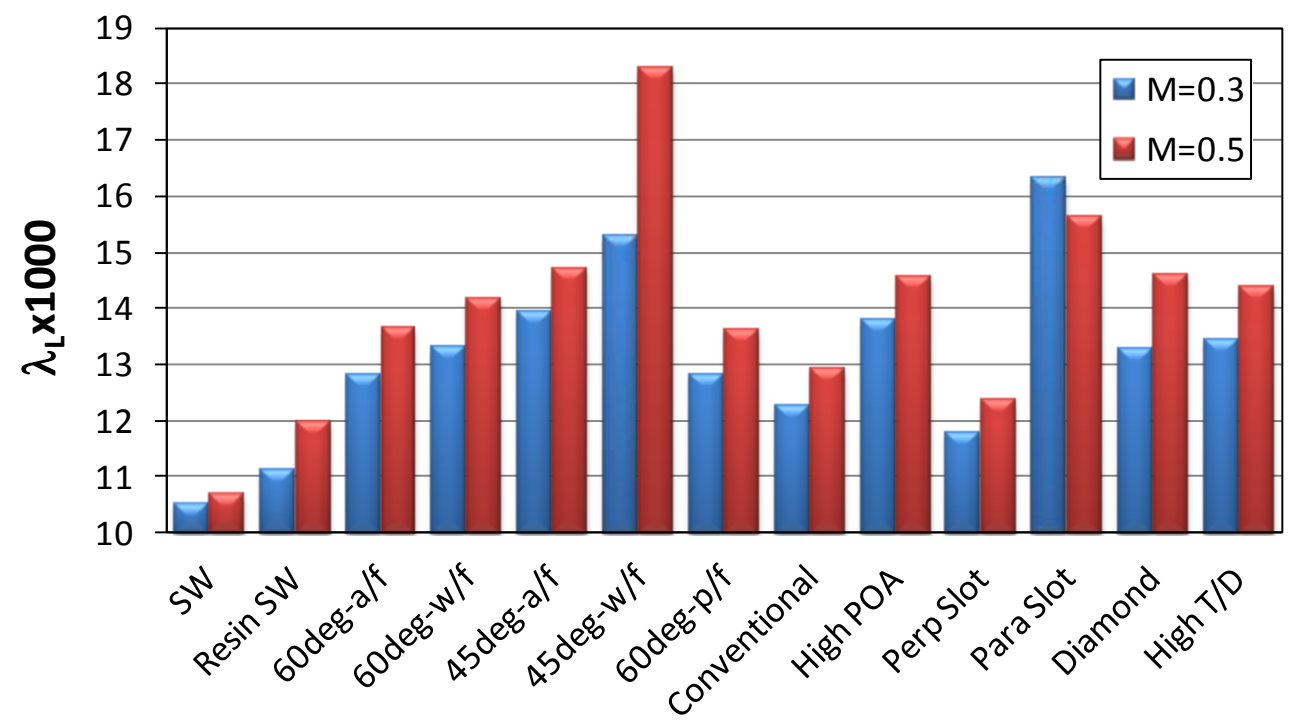

Figure 8. Liner resistance factor $\left(\lambda_{L}\right) \times 1000$ for $M=0.3$ and 0.5 , no sound.

Generally, $\lambda_{\mathrm{L}}$ increases with increasing Mach number except for the Parallel Slot configuration which saw a slight decrease. All configurations produce a drag penalty relative to the Resin SW. Angling the perforations into the flow, 
especially for the shallower 45 deg configuration, proved very detrimental to liner drag. Overall, many of the configurations tested result in increased drag relative to the Conventional perforate design. Only the Perpendicular Slot shows a reduction but still carries a penalty relative to both smooth wall configurations. The Resin SW result is also higher than the aluminum SW for both Mach numbers indicating increased surface roughness for the printed sample.

Figure 9 is a plot of the percent difference of the liner resistance factor $\left(\lambda_{\mathrm{L}}\right)$ relative to the Resin SW configuration. None of the angled, round hole designs perform better than the straight-hole Conventional configuration. Of those, facesheets with perforations oriented with the flow are measureably worse than their counterparts with perforations oriented against the flow. Note that for the $45 \mathrm{deg}$ angle, the penalty more than doubled for $\mathrm{M}=0.5$. The High T/D design produces a $20 \%$ increase that was nearly double the result for the Conventional configuration. Of all the alternate configurations tested, only the Perpendicular Slot design performs better than the Conventional with a $\Delta \lambda_{\mathrm{L}}$ approximately $50 \%$ lower and penalty relative to the Resin SW of $\sim 5 \%$. The High POA and Parallel Slot cases show penalties of over $20 \%$ and 30\%, respectively, indicating that small changes in the perforate configuration can have a large impact on $\lambda_{\mathrm{L}}$.

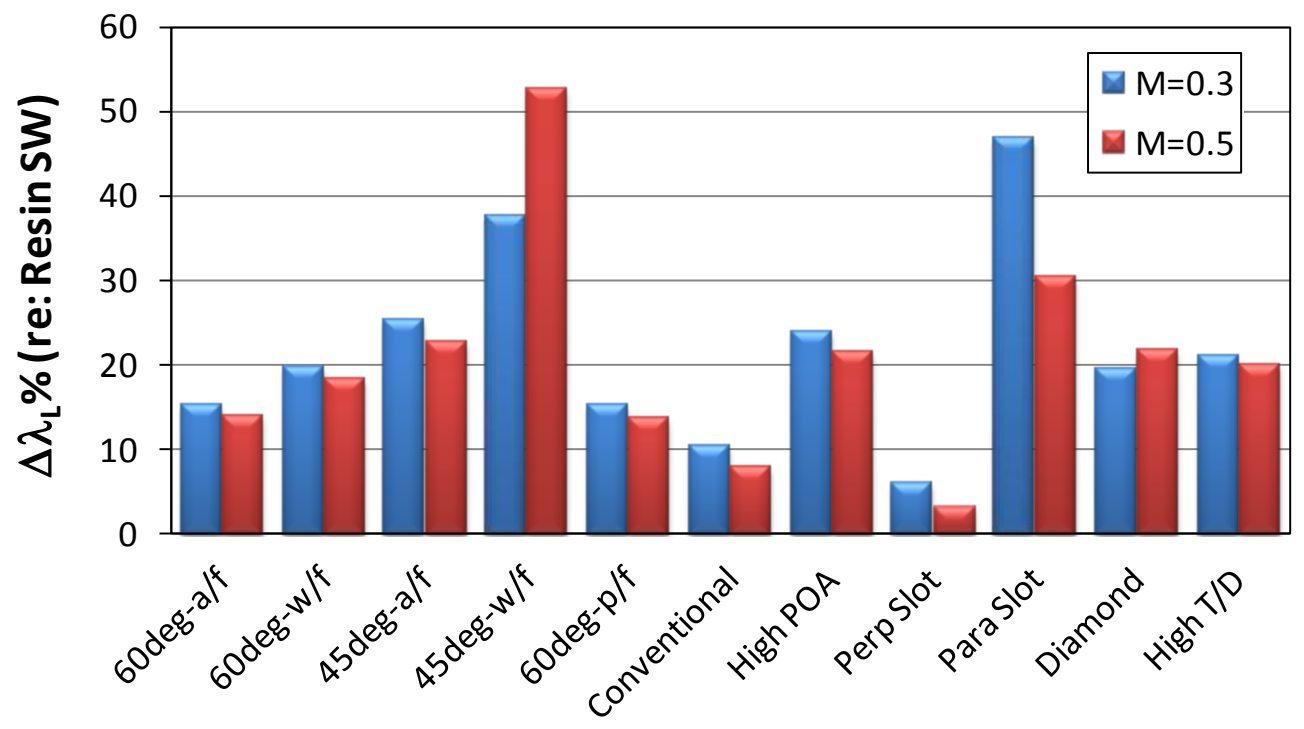

Figure 9. Percent difference in $\lambda_{L}$ (relative to Resin SW) for $M=0.3$ and 0.5 , no sound.

\section{B. Effects of Acoustic Excitation on drag}

Each liner configuration was evaluated in the presence of acoustic tonal excitation at a target total SPL of 140 and $150 \mathrm{~dB}$. Frequencies ranged from $400 \mathrm{~Hz}$ to $3000 \mathrm{~Hz}$, in $200 \mathrm{~Hz}$ increments. It was postulated prior to the test that the oscillatory motion of fluid through the facesheet perforations would affect the measured resistance factor. Expectations of significant variation in $\lambda_{\mathrm{L}}$ at or near the frequencies of resonance $(\sim 1700 \mathrm{~Hz}$ for these configurations) and anti-resonance were also postulated. Figure 10 shows resistance factor spectra for the two Mach numbers and SPL's. From these plots, several trends emerge. The resistance factor of the liner varies with frequency. The variation increases with increasing SPL and decreases with increasing Mach number. Thus, there exist combinations of Mach number and SPL for which $\lambda_{\mathrm{L}}$ is relatively invariant with frequency. This behavior is desired from a design perspective since it can eliminate frequency as an optimization variable. For flow speeds of Mach 0.5 , even the $150 \mathrm{~dB}$ level results in variations only on the order of $10 \%$, significantly less than that observed for the Mach 0.3 cases. The expectation of some discernable effect of resonance is not borne out by the data for any Mach number or SPL. In fact, the most notable feature of the spectra is the dip in all the $150 \mathrm{~dB}$ results at $1200 \mathrm{~Hz}$. The current design of the GFIT acoustic speaker array and anechoic terminations seems to inhibit efficient acoustic energy transmission from the loudspeakers into the duct, limiting the maximum SPL at that frequency to approximately $141 \mathrm{~dB}$. This level is substantially less than the other frequencies tested and, thus, produces less of an effect on the liner resistance factor. 
$\mathbf{M}=\mathbf{0 . 3}$
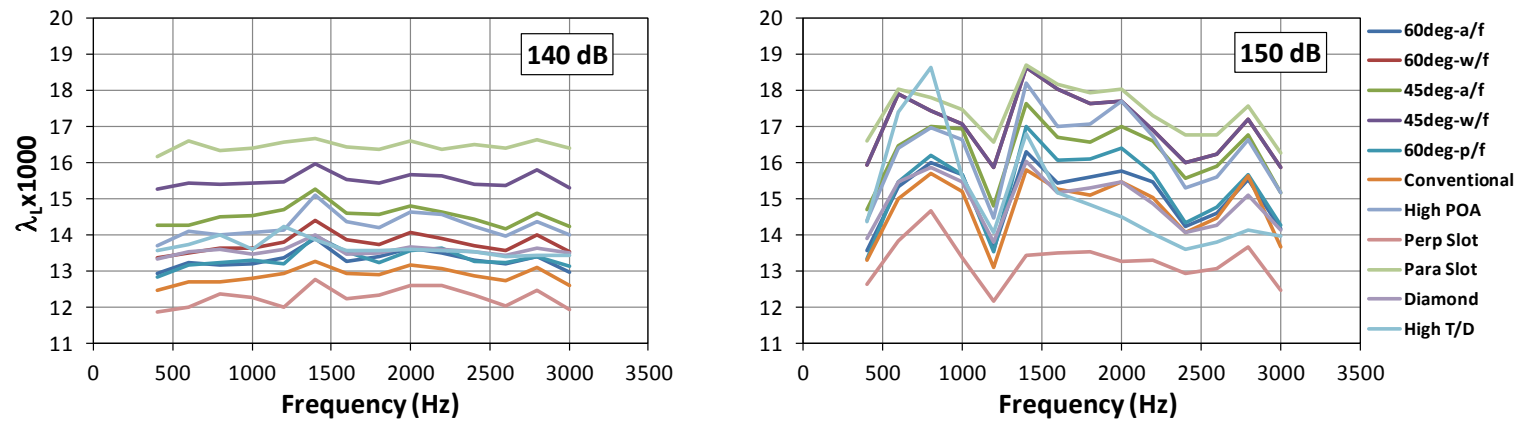

\section{$\mathrm{M}=\mathbf{0 . 5}$}
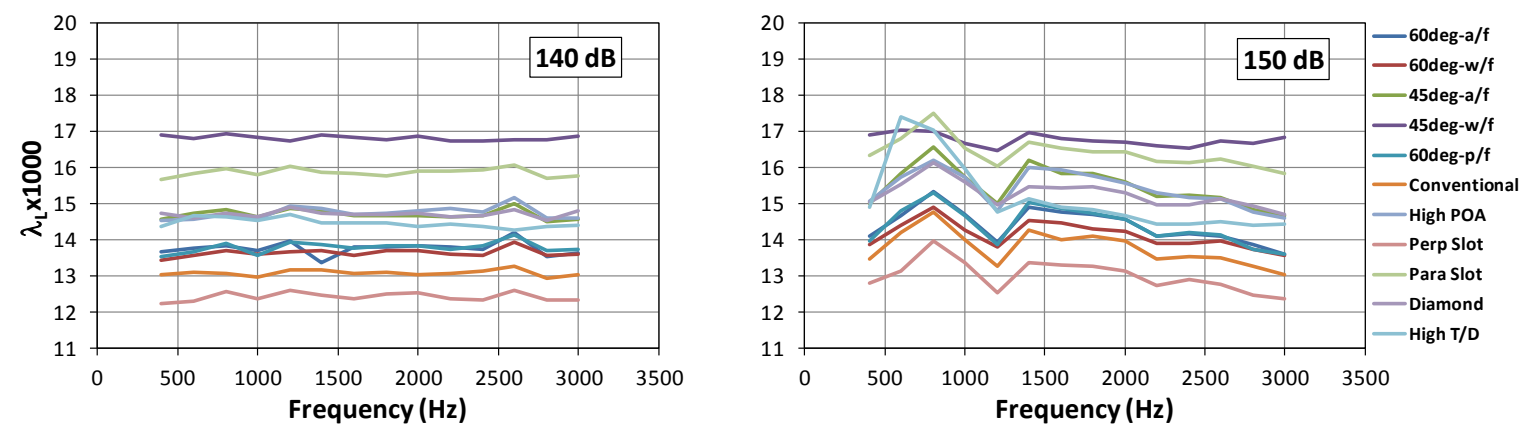

Figure 10. Variation of resistance factor with acoustic excitation.

\section{Acoustic Measurements}

For each liner configuration, full acoustic pressure profiles were acquired at $M=0.3$ and 0.5 for the purposes of impedance eduction and evaluation of attenuation performance. As noted before, there are certain frequencies where $150 \mathrm{~dB}$ was not achieved, but at least $145 \mathrm{~dB}$ is achieved for the majority of those cases. For that reason, the acoustic results presented below are for the $\mathrm{M}=0.5,140 \mathrm{~dB}$ cases. These educed impedance spectra are compared to determine if variations in perforate geometry and orientation have significant impact on the liner acoustic performance relative to the Conventional configuration. Ideally, variations in perforate shape would have minimal acoustic effect ${ }^{8}$, thereby allowing for selection based on drag performance. Results from the High T/D sample are omitted for the sake of clarity since the configuration's significantly higher resistance values, relative to the other facesheets, would expand the scale and obscure details of the other spectra.

Figure 11 shows the normalized impedance spectra (all impedances are normalized by $\rho c$ ) for each configuration. For resistance, the differences between facesheets are less than $\sim 0.7 \rho c$ at all frequencies and variation in reactance is generally less than $0.5 \rho c$. Resonance for these configurations varies between approximately 1700 and $2000 \mathrm{~Hz}$ with the majority clustered near $2000 \mathrm{~Hz}$. The Perpendicular Slot and Diamond exhibit a lower resonance near $1700 \mathrm{~Hz}$. The shape of the impedance spectra for all of the liners are similar (note the expanded scale for the resistance spectra). These results imply that a low-drag design could be tailored to closely match the impedance spectra of a higher-drag, conventional design. Figure 12 focuses on a comparison of the impedance spectra for the Conventional and low-drag Perpendicular Slot configurations. The educed resistance and reactance spectra compare very favorably and and imply that acoustic performance does not have to be sacrificed in order to reduce liner drag. 

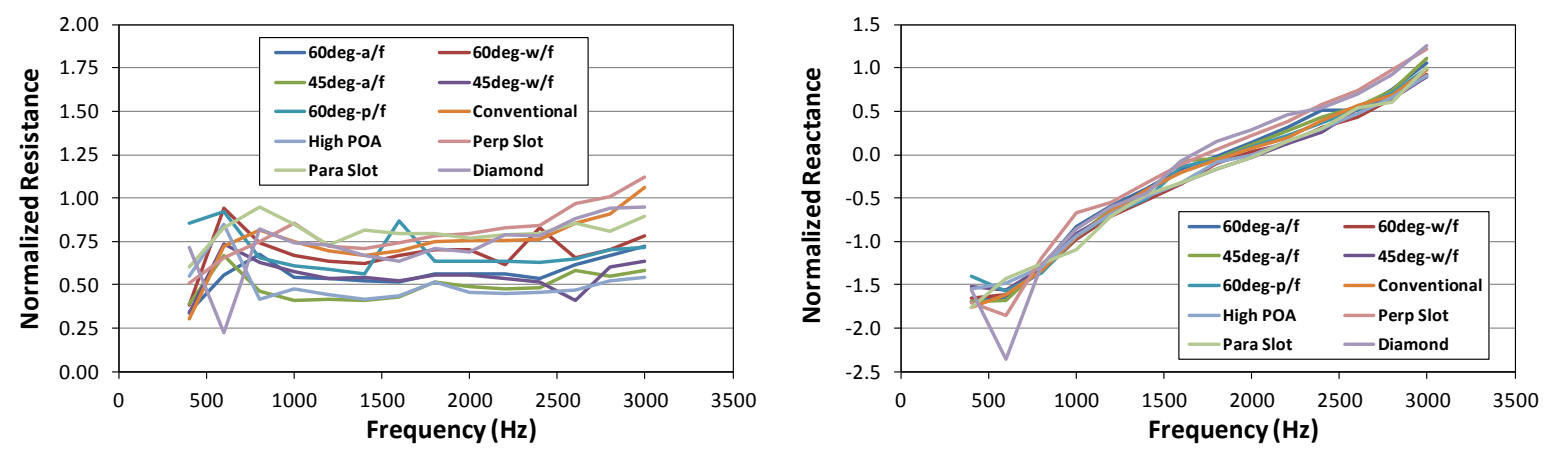

Figure 11. Normalized impedance spectra for each configuration, $M=0.5,140 \mathrm{~dB}$.
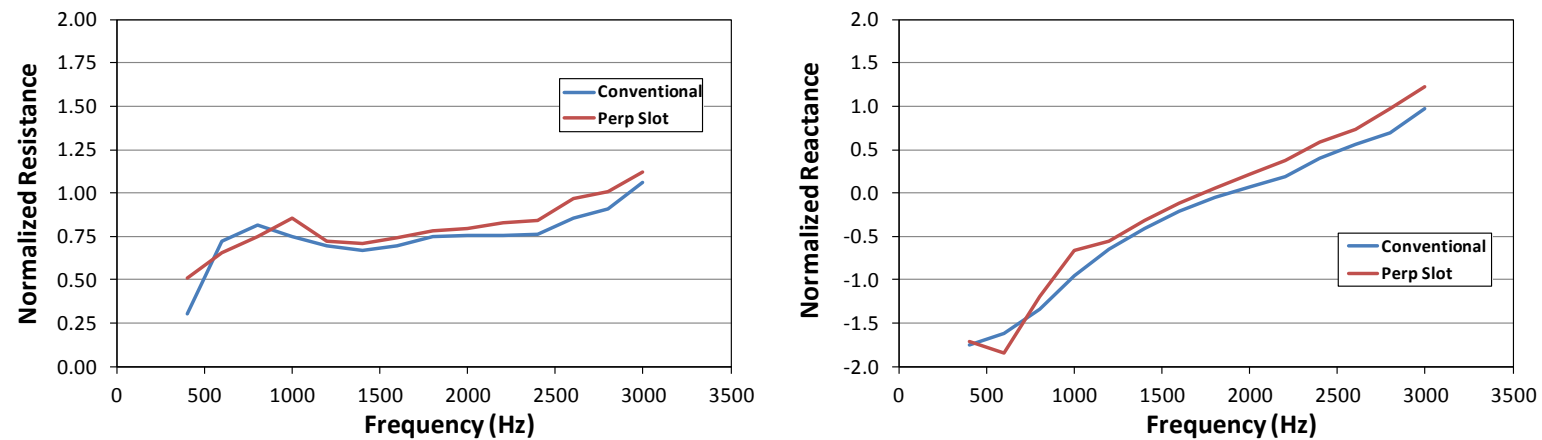

Figure 12. Comparison of normalized impedance spectra, Conventional (round hole) vs. Perpendicular Slot configurations, $M=0.5,140 \mathrm{~dB}$.

This conclusion is further supported by the data shown in Fig. 13 where the acoustic pressure profiles are plotted for selected frequencies bracketing resonance. The SPL from each of the 95 GFIT microphones are plotted against their axial location in the duct. $X=0$ corresponds to a point $203.2 \mathrm{~mm}$ upstream of the liner leading edge. The profiles for each frequency compare well indicating that both designs have very similar acoustic performance.

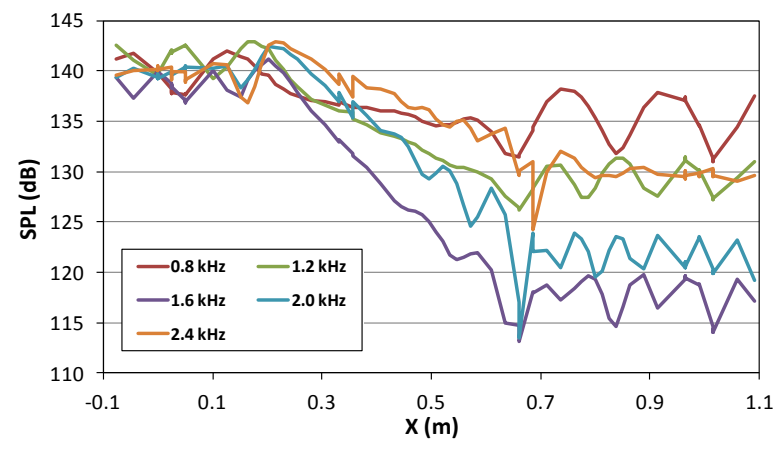

Conventional

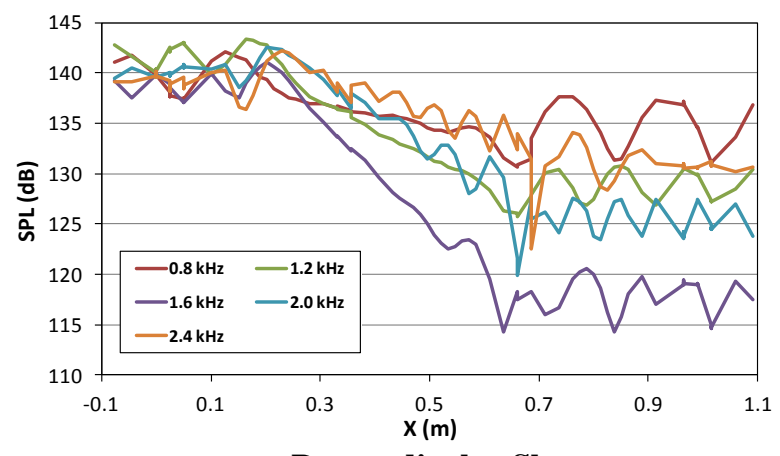

Perpendicular Slot

Figure 13. Comparison of axial attenuation, $M=0.5,140 \mathrm{~dB}$.

Another method of evaluating liner performance is to compare the levels of attenuation achieved by the various configurations. This calculation gives a gross estimate of the effect these variations in perforate geometry have on the overall acoustic performance of the liner. For this study, a simple estimate of attenuation was calculated using 
the difference in sound pressure levels measured at the leading and trailing edges of the duct $(203.2 \mathrm{~mm}$ upstream of liner leading edge and $355.6 \mathrm{~mm}$ downstream of liner trailing edge, respectively). Such a result does include the effects of reflections from these edges and the influence of the duct termination. However, it provides a useful quantity for comparison. Figure 14 shows the results of this calculation and confirms that for much of the frequency range tested, the differences in perforate geometry do not significantly impact the resulting liner attenuation.

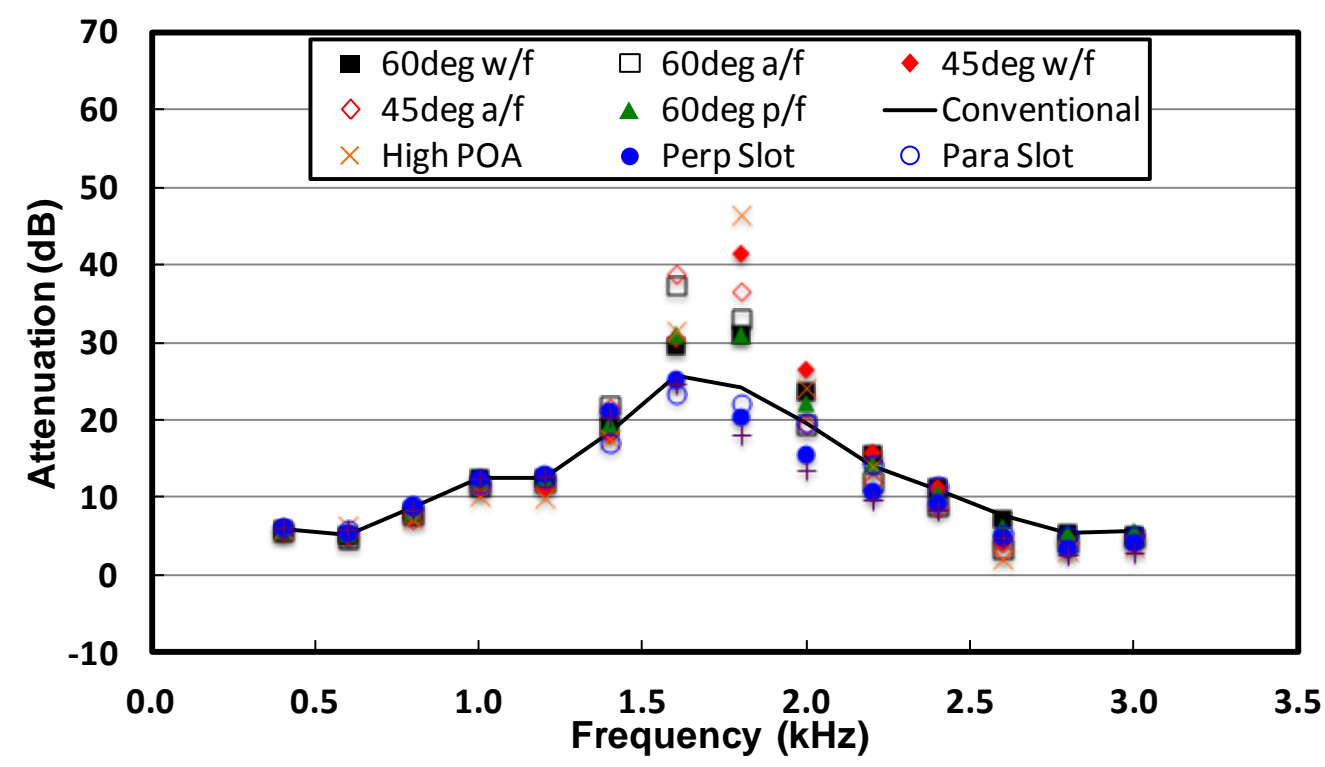

Figure 14. Comparison of overall liner attenuation, $M=0.5,140 \mathrm{~dB}$.

For frequencies near resonance (approximately $1900 \mathrm{~Hz}$ for this liner geometry), the variation between configurations grows to as much as $+20 /-5 \mathrm{~dB}$ relative to the Conventional perforate. Figure 15 presents this data using the average loss in attenuation (in $\mathrm{dB}$ ), relative to the Conventional configuration, across the frequency range. Using this metric, it can be shown that the Perpendicular Slot, Parallel Slot and Diamond perforates perform slightly worse overall than the Conventional configuration. Several perforates have average attenuations better than Conventional, but all the variation is within $+/-1.5 \mathrm{~dB}$ indicating that the change in perforate geometry has little effect on overall liner performance.

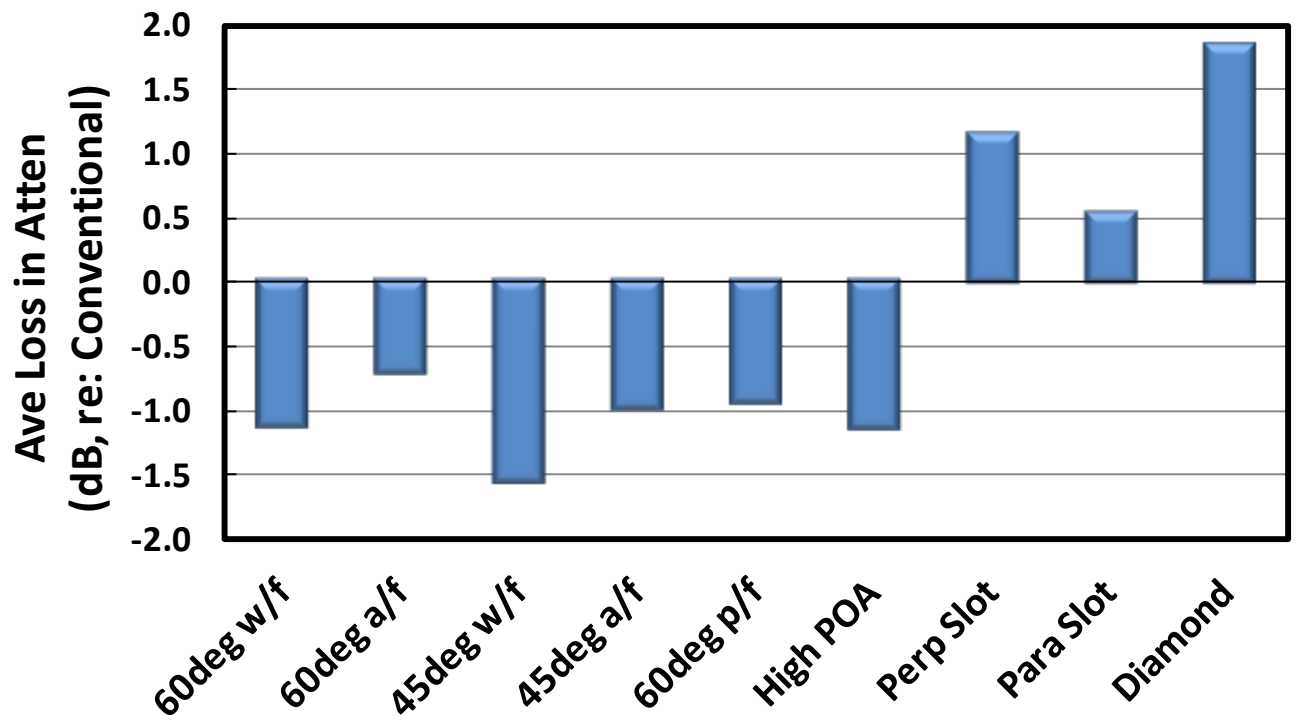

Figure 15. Relative attenuation loss, $M=0.5,140 \mathrm{~dB}$. 


\section{Concluding Remarks}

A set of liner facesheets were tested in the NASA Langley GFIT to assess their drag relative to a smooth wall and a conventional straight, round-hole perforate. Several observations were made:

1. Adding angle/skew to a conventional, round-hole perforate configuration increased facesheet drag, especially for holes angled at $45 \mathrm{deg}$.

2. Angling the holes with the flow increased the measured resistance factors more than angling against the flow. The shallower $45 \mathrm{deg}$ case with this orientation produced the most drag of all configurations tested.

3. The Perpendicular Slot configuration produced the lowest drag and was the only perforate design that performed better than the Conventional design.

4. Changes to the perforate geometry caused some variation to the normalized resistance spectra, on the order of $0.5 \rho c$.

5. Changes to perforate geometry caused minimal variation to the normalized reactance spectra. Resonant frequencies clustered aroung $2000 \mathrm{~Hz}$ for most cases. The Diamond and Perpendicular Slot liners resonated near $1700 \mathrm{~Hz}$.

6. Compared to the Conventional configuration, differences in overall attenuation between the perforate geometries are small.

7. Acoustic liner performance need not be sacrificed to reduce liner drag. The acoustic characteristics of the identified low-drag perforate were sufficiently close to the conventional design that minor geometry changes would be all that are required to recover any lost performance.

\section{Acknowledgments}

The authors would like to thank Carol Harrison of the NASA Langley Structural Acoustics Branch for her efforts in collecting the experimental data. Funding for this effort was provided under NASA's Advanced Air Transport Technology Project for the Advanced Air Vehicles Program and by the Environmentally Responsible Aviation Project for the Integrated Aviation Systems Program.

\section{References}

${ }^{1}$ Tam, Christopher K. W., Pastouchenko, Nikolai N., Jones, Michael G., Watson, Willie R., "Experimental Validation of Numerical Simulations for an Acoustic Liner in Grazing Flow," AIAA Paper 2013-2222, 19 ${ }^{\text {th }}$ AIAA/CEAS Aeroacoustics Conference, May 2013.

${ }^{2}$ Drouin, M. K.,Gallman, J. M., and Olsen, R.F., “Sound Level Effect on Perforated Panel Boundary Layer Growth,” AIAA Paper 2006-2411, May 2006.

${ }^{3}$ Thomas, R. H., Burley, C. L., Lopes, L. V., Bahr, C. J., Gern, F. H. and Van Zante, D. E., "System Noise Assessment and the Potential for a Low Noise Hybrid Wing Body Aircraft with Open Rotor Propulsion," AIAA Paper 2014-0258, January 2014.

${ }^{4}$ Nikuradse, J., "Laws of Flow in Rough Pipes," NACA TM-1292, November 1950.

${ }^{5}$ Howerton, Brian M., and Jones, Michael G., "Acoustic Liner Drag: A Parametric Study of Conventional Configurations," AIAA Paper 2015-2230, $21^{\text {st }}$ AIAA/CEAS Aeroacoustics Conference, AIAA Aviation 2015, June 2015.

${ }^{6}$ Hwang, D.P., "A proof of concept experiment for reducing skin friction by using micro-blowing technique", AIAA paper 97-0546, 1997.

${ }^{7}$ Watson, W. R., and Jones, M. G., "A Comparative Study of Four Impedance Eduction Methodologies Using Several Test Liners," AIAA Paper 2013-2274, 19 th AIAA/CEAS Aeroacoustics Conference, May 2013.

${ }^{8}$ Ahuja, K. K., and Gaeta, R. J., “Active Control of Liner Impedance by Varying Perforate Orifice Geometry,” NASA CR2000-210633, December 2000. 